\title{
External Disturbances Rejection by Differential Single-Mass Vibratory Gyroscope
}

\author{
Valerii V. Chikovani, Olha A. Sushchenko, Hanna V. Tsiruk \\ National Aviation University \\ Kosmonavt Komarov avenue, Kyiv, 03058, Ukraine \\ v_chikovani@nau.edu.ua, olha_sushch@nau.edu.ua, hanna.tsiruk@nau.edu.ua
}

\begin{abstract}
Vibratory gyroscopes are now most applicable for such intelligent systems as drones, for motion stabilization, robots for accurate positioning of end-effectors, virtual reality systems to change image orientation with turn of a head and many others. During motion these systems can be exposed to mechanical shocks and vibrations. To provide required accuracy, working in such environmental conditions, gyroscopes shall have property of robustness to operating disturbances. This paper proposes differential mode of operation for single-mass vibratory gyroscope as a new operating mode that has higher rejection factors for different external disturbances like shocks and vibrations allowing meeting the requirements of many important applications in intelligent systems. Test results presented in this paper show excellent disturbance rejection properties of differential mode of operation in comparison to well-known rate mode. Despite excellent disturbance rejection results have been obtained for non MEMS gyro, the same results can certainly be obtained for MEMS gyro, too.
\end{abstract}

Keywords: differential vibratory gyroscope; shock rejection factor; vibration sensitivity

\section{Introduction}

Coriolis vibratory gyro (CVG) is one of the chronologically latest gyroscopic technology appeared in the world market in the 90s of the previous century. This technology for the sufficiently short time spread out all over the world mainly due to its micro-miniature variant based on micro-electro-mechanical system (MEMS). Vibratory gyros in MEMS implementation have many applications in intellectual systems such as drone for motion stabilization and attitude control, robots for positioning of end-effectors and indoor and outdoor navigation, virtual reality helmet to change image orientation in accordance to the turning of a head and many others including medical and space applications.

There are two well-known modes of CVG operation:

1) Closed loop mode or rate mode [6] [10] [11] [22] where primary stable amplitude standing wave excited in a vibrating structure at one of its resonant frequencies is retained in the vicinity of the drive electrode by the control forces. 
Gyro rotation produces Coriolis forces exciting a secondary standing wave that is compensated for by applying the control forces to keep the standing wave at the stable position in the vicinity of the drive electrode. The amplitude of the control signal that compensates for the Coriolis force is proportional to the angle rate.

2) Whole angle or rate-integrating mode [4] [10] [11] [12] when under gyro rotation, Coriolis forces provide transformation of vibration energy from primary to secondary modes and vice versa. Quadrature signal is the only one that is compensated for, to reduce CVG errors [11]. In this case, gyro rotation angle is proportional to standing wave rotation angle caused by Coriolis forces.

There is also an open loop mode of operation, where a standing wave, excited in a resonator, is not controlled by any forces, except the drive force [6]. This mode of operation is rarely used in practice, because of a large bias instability and a low dynamic accuracy [10].

The first, rate, mode of CVG operation is most popular one because of lower influence of manufacturing imperfections, lower noise when measuring small angle rate and it has an acceptable bandwidth for most applications. The second, rate-integrating, mode can have extremely high dynamic range, high bandwidth and very stable scale factor, but it has higher sensitivity to manufacturing imperfections.

Comparatively recent investigations [17] [18] [19] [20], resulted in the third, differential, mode of $\mathrm{CVG}$ operation which complements first two modes possessing additional capabilities for suppression of external disturbances. The differential mode of operation can also be implemented in well-known tuning fork design of MEMS or non-MEMS CVGs, because they have two anti-phase vibrating resonators and they can reject disturbances, for example shocks [3] [14] by subtracting signals coming from two resonators, or can survive after very high shocks [13]. In spite of tuning fork and other multi-mass resonator MEMS designs have external disturbance rejection properties they cannot effectively be used in practical applications because their rejection factor is high enough for small shocks and capability to survive does not provide measurements during shock. This is because different resonators have no equal parameters such as $Q$ factor, rigidity, resonant frequency and, as a consequence, they have different responses to the same external disturbance.

The proposed differential mode of operation can be implemented in single-mass resonator CVG by keeping a standing wave between the electrodes by applying two stable amplitude control voltages on $\underline{X}$ and $\underline{Y}$ drive electrodes. In this case two magnitudes of angle rates with opposite signs can be picked up from $\underline{X}$ and $\underline{Y}$ sense electrodes. The resulting angular rate can be obtained by subtraction of the two measurement channel signals. Rejection factor in this case is increased because responses of the two ( $\underline{X}$ and $\underline{Y}$ ) channels of the single-mass resonator are much closer to each other, than in case of different mass resonators. 
At proper angular alignment of standing wave $\theta^{*} \neq \underline{m} \pi / 4, \underline{m}=0,1 \ldots$, cross damping bias component is compensated for. This can be reached at standing wave angular position that equalizes $\underline{X}$ and $\underline{Y}$ measurement channel scale factors $\underline{S F_{x}}$ and $\underline{S F_{y}}$ [18].

CVGs defer from other gyroscope technologies by that all practically interesting, which we are numbered here as the first, second and third modes of operation can be implemented in a single triple-mode vibratory gyroscope with automatic switching from one mode to other [21]. It should be noted that dual mode CVG (the first and second ones) have been implemented and described in [7]. The latter gives undeniable advantages of CVGs over competitive technologies, ring laser and fiber optic gyros, in terms of dynamic range, bandwidth, dynamic error in measuring high angle rate, lower noise in measuring small angle rate and reliability [8]. For example, under measuring of small angle rate it is advisable to operate in the rate mode, since the measurement errors are mainly determined by noise and bias drift which can be lower, than that of for rate-integrating mode of operation. Under measuring of high angle rate (more than $500 \mathrm{deg} / \mathrm{s}$ ) or higher, it is advisable to operate in the second, rate-integrating, mode of operation since the measurement errors are mainly determined by multiplicative error $\Delta \Omega$ caused by scale factor uncertainty, $\Delta \underline{S F}, \Delta \Omega=\Delta \underline{S F^{*}} \Omega$. Scale factor for rate-integrating mode of operation is a stable constant (Bryan coefficient). It can reach $35 \mathrm{ppm}$ and its dynamic range is up to $7 \times 10^{3} \mathrm{deg} / \mathrm{s}$ and more for even low-cost gyros [2].

When gyro is operating under high external disturbances (shocks, vibrations, magnetic fields or others), it is advisable to operate in the third (differential) mode of operation, since this mode of operation for single-mass CVG has higher disturbance rejection factor, than the first and second modes of the same CVG and the third mode for a multi-mass CVG. Switching from one mode to another can be implemented in accordance with changing environmental conditions using intelligent algorithm based on, for example, fuzzy logic.

This paper presents test results showing excellent disturbance rejection properties of differential mode of operation for single-mass CVG in comparison to rate mode under action of external mechanical shocks and vibrations.

\section{Differential Mode of Operation}

Because rate and rate-integrating modes of CVG operation are well-known [4] [6] [10] [11] [12] [22] let's shortly describe the differential mode of operation for single-mass resonator gyro.

In differential CVG standing wave is located between the electrodes so that wave angle $\theta \neq \underline{m} \pi / 4, \underline{m}=0,1,2 \ldots$, that is standing wave oscillation direction is not coincident with any of electrodes, as depicted, in Figure 1. 
In this case CVG output signals in voltages, $\underline{z}_{\underline{x}}$ and $\underline{z}_{y}$, in the differential mode of operation, under nulling quadrature signal, can be written as follows [20]

$$
\begin{gathered}
-2 k \Omega D_{y} \operatorname{tg} 2 \theta+D_{x} d_{x x}+d_{x y} D_{y} \operatorname{tg} 2 \theta=z_{x} \\
2 k \Omega D_{x} \operatorname{ctg} 2 \theta+D_{y} d_{y y}+d_{x y} D_{x} \operatorname{ctg} 2 \theta=z_{y}
\end{gathered}
$$

where,

$$
\begin{aligned}
& d_{x x}=\left[\frac{2}{\tau}+h \cos 2\left(\theta-\theta_{\tau}\right)\right] ; h=\Delta\left(\frac{1}{\tau}\right)=\frac{1}{\tau_{1}}-\frac{1}{\tau_{2}} ; \\
& \frac{2}{\tau}=\frac{1}{\tau_{1}}+\frac{1}{\tau_{2}} ; d_{y y}=\left[\frac{2}{\tau}-h \cos 2\left(\theta-\theta_{\tau}\right)\right] ; \\
& d_{x y}=h \sin 2\left(\theta-\theta_{\tau}\right)
\end{aligned}
$$

$\underline{D}_{\underline{x}}$ and $\underline{D}_{y_{-}}$are transformation coefficients of resonator deformations into voltage for $\underline{X}$ and $\underline{Y}$ electrodes, respectively; $\tau_{1}, \tau_{2}$ are time constants along resonator damping pricipal axis, $\tau_{1}=\tau_{\min }$ which is located under angle $\theta_{\tau}$ relative to direction of standing wave oscilation and $\tau_{2}=\tau_{\max }$ which is located under angle $45 \mathrm{deg}$ to the direction of $\tau_{\min }$ axis, $\tau_{\max }$ axis not shown in figure $1 ; k$ is Bryan coefficient.

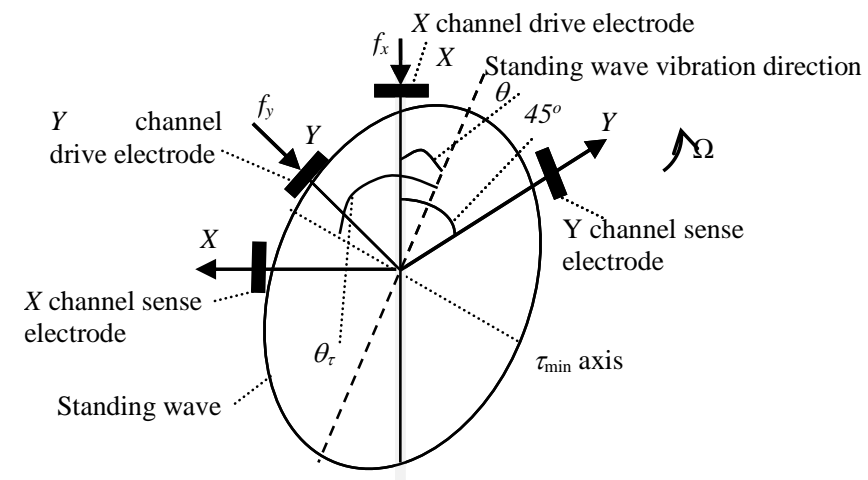

Figure 1

Standing wave position under angle $\theta \neq \underline{m} \pi / 4, \underline{m}=0,1,2 \ldots$, in differential mode of CVG operation

As can be seen from equations (1) there are two $\underline{X}$ and $\underline{Y}$ measurement channels, with negative $-\Omega$ and positive $\Omega$ angle rates, respectively. Thus, control system that retains the standing wave between electrodes implements differential mode of operation for single-mass resonator CVG. As can also be seen from (1), $\underline{X}$ and $\underline{Y}$ channels scale factors $\underline{S F_{\underline{x}}}, \underline{S F_{y}}$ and biases $\underline{B}_{\underline{x}}, \underline{B}_{\underline{y}}$ are dependent on angle $\theta$ as follows

$$
\begin{array}{ll}
S F_{x}=2 k D_{y} \tan 2 \theta & B_{x}=D_{x} d_{x x}+d_{x y} D_{y} \tan 2 \theta \\
S F_{y}=2 k D_{x} \cot 2 \theta & B_{y}=D_{y} d_{y y}+d_{x y} D_{x} \cot 2 \theta
\end{array}
$$


One can choose angle $\theta$, from application point of view, such that $\underline{B}_{\underline{x}}=\underline{B}_{y}$, or $\underline{S F_{x}}=\underline{S F_{y}}$, both of these angles are different and close to $22.5 \mathrm{deg}$ [21]. To effectively implement differential mode of operation it is advisable to align standing wave under angle $\theta^{*}$ at which $\underline{S F_{x}}=\underline{S F_{y}}$, so using (2) the following relationship can be written down

$$
D_{y} \tan 2 \theta^{*}=D_{x} \cot 2 \theta^{*} \text { or } \theta^{*}=\frac{1}{2} \arctan \sqrt{\frac{D_{x}}{D_{y}}}=\frac{1}{2} \arctan \sqrt{\frac{S F_{y}}{S F_{x}}}
$$

When standing wave angle is $\theta^{*}$, half sum and half difference of the $\underline{X}$ and $\underline{Y}$ measurement channels can be represented as follows [19]

$$
\begin{aligned}
& \left(z_{x}-z_{y}\right) / 2=-S F_{d} \Omega+D_{y} d_{y y}-D_{x} d_{x x} \\
& \left(z_{y}+z_{x}\right) / 2=D_{y} d_{y y}+D_{x} d_{x x}+2 d_{x y} \sqrt{D_{x} D_{y}} \\
& S F_{d}=2 k \sqrt{D_{x} D_{y}}
\end{aligned}
$$

where, $\underline{S F_{d}}$ is a scale factor of differential CVG, when $\theta=\theta^{*}$. As can be seen from (4) difference signal of $\underline{X}$ and $\underline{Y}$ measurement channels has no damping cross coupling term $\underline{d}_{x y}=\underline{h \sin } 2\left(\theta-\theta_{\tau}\right)$, and sum of these channels has no angle rate, but contains current information about main bias components that can supposedly be used for their on-line estimation. In case when angle $\theta \neq \theta^{*}$, and $\theta \neq \underline{m} \pi / 4, \underline{m}=0,1$, $2, \ldots$, then difference and sum of the channels have different scale factors,

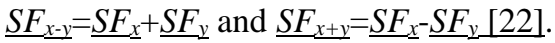

Differential CVG control system block diagram operating in differential mode of operation under command signal $\theta_{\text {comm }}=-\theta *$ is presented in Figure 2. It should be noted that when $\theta_{\text {comm }}=0$ it operates in the rate mode and when connection of $\theta_{\text {real }}$ signal to proportional and integral (PI) controller is open, it operates in rateintegrating mode [21].

In the differential mode of operation two drive signals $\underline{X}_{\underline{i n}}$ and $\underline{Y}_{\underline{i}}$ excite standing wave so that it is located between the electrodes at an angle $\theta=\theta^{*}$. Frequency tracking subsystem presented in Figure 2 is based on phase lock loop (PLL) implemented in digital form and consists of phase detector, loop filter and voltage controlled oscillator [1] (numerically controlled oscillator in digital case). CVG uses PI controllers to compensate for Coriolis force and quadrature signals.

Figure 3 demonstrates $\underline{X}, \underline{Y},(\underline{X}-\underline{Y}) / 2$ and $(\underline{X}+\underline{Y}) / 2$ signals under measuring of constant angle rates $\pm 30 \mathrm{deg} / \mathrm{s}$ after each channel bias subtraction. For the gyro being under test angle $\theta^{*}=25.067 \mathrm{deg}$. Figure 3 shows absence of angle rate in the $(\underline{X}+\underline{Y}) / 2$ channel that can help one to see current measurement error components.

The next sections present test results that allow us to quantitatively determine disturbance rejection factors of differential mode of operation for single-mass ring type resonator $\mathrm{CVG}$ in comparison to rate mode of the same $\mathrm{CVG}$, when disturbances are external mechanical shocks and vibrations. 


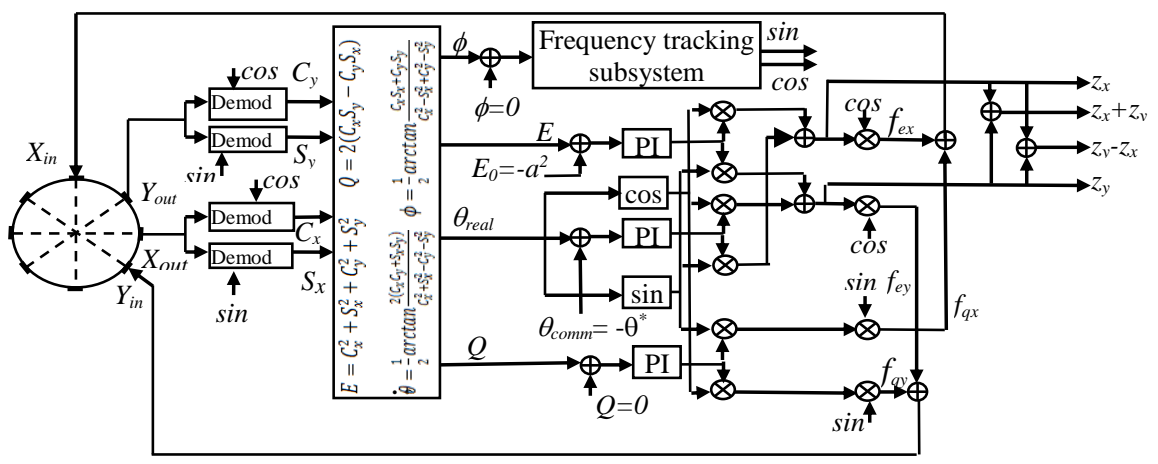

Figure 2

CVG control system block diagram operating in differential mode of operation

It should be noted that in all tests dampers, screens and other disturbance protection means did not use, so all rejection factors and external disturbance sensitivities have been determined for exposed differential CVG.

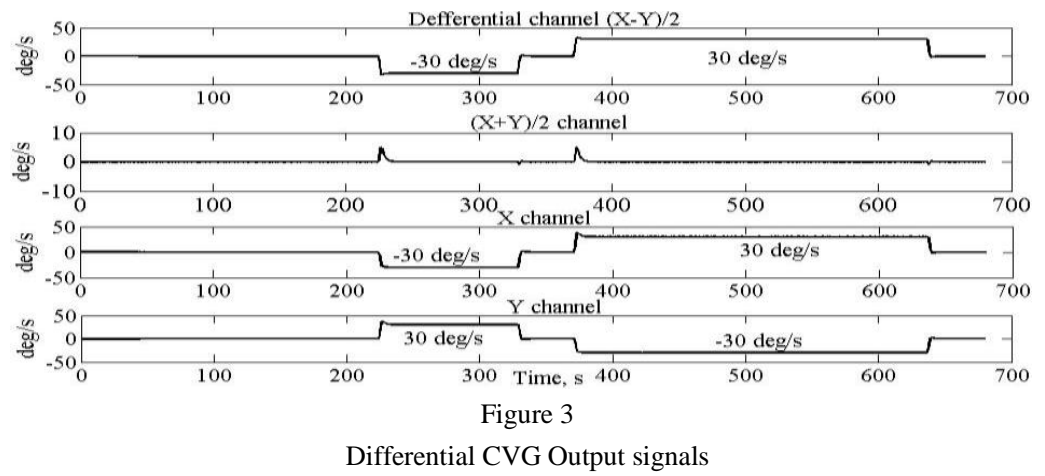

\section{Shock Rejection Factor}

Since each of the two $\underline{X}$ and $\underline{Y}$ measurement channels operate, in essence, in the rate mode and their difference presents differential mode signal, then ratio of one of the $\underline{X}$ and $\underline{Y}$ channel signals to half difference one, $(\underline{X}-\underline{Y}) / 2$, will determine disturbance rejection factor $R_{S}$ of CVG rate mode as compared with differential one. Ratio will be determined after bias subtraction for each of the channels. Thus, rejection factor for external mechanical shock will be calculated as follows

$R_{s}=\min \left\{\frac{\operatorname{mean}\left(\operatorname{abs}\left(X_{i}\right)\right)}{\operatorname{mean}\left[\operatorname{abs}\left(\left(X_{i}-Y_{i}\right) / 2\right)\right]}, \frac{\operatorname{mean}\left(\operatorname{abs}\left(Y_{i}\right)\right)}{\operatorname{mean}\left[\operatorname{abs}\left(\left(X_{i}-Y_{i}\right) / 2\right)\right]}\right\}, i=1 \ldots 5(5)$

where, $\underline{X}_{\underline{i}}$ and $\underline{Y} \underline{\underline{i}}$ are peak values of $\underline{X}$ and $\underline{Y}$ channel responses for an $\underline{t}^{\text {th }}$ shock. 
Bias sensitivity coefficient $\underline{S}_{g}$ to shock or vibration acceleration $\underline{A}_{g}$ will be calculated as a ratio

$$
S_{g}=\operatorname{mean}\left[a b s\left(X_{i}-Y_{i} / 2\right)\right] / A_{g}, i=1 \ldots 5
$$

\subsection{Shocks along Input Axis}

Figure 4 shows superposed $\underline{X}$ and $\underline{Y}$ channel signals of differential CVG after exposure to low amplitude mechanical shock (less, than $5 \mathrm{~g}$, where $\mathrm{g}$ is free fall acceleration) along gyro input axis (IA). As can be seen from figure $4 \underline{X}$ and $\underline{Y}$ channel responses are almost equal to each other, so differential channel response is close to zero. The latter means that rejection factor to low shock is very high, as has also been demonstrated in [15] for tuning fork MEMS gyro. There is not a noticeable angle rate that usually presents in high $g$ shocks and, as a consequence, peak values almost coincide. The same result can be obtained for low amplitude lateral shock (perpendicular to IA).

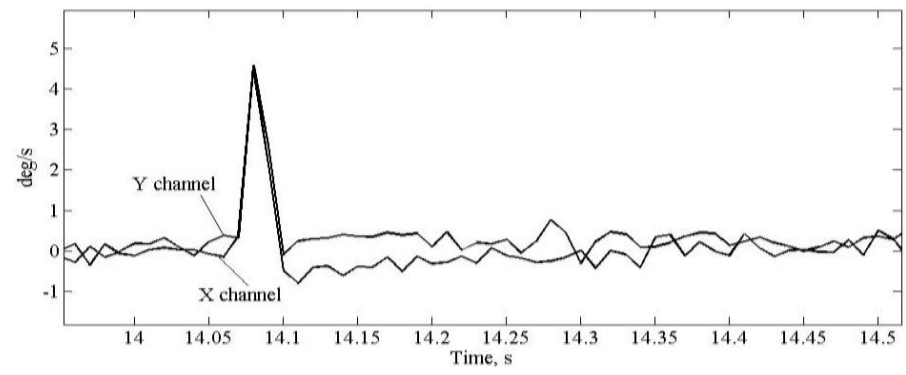

Figure 4

$\underline{X}$ and $\underline{Y}$ channels superposed responses to small shock

When shock increases to $20 \mathrm{~g}$ of $2 \mathrm{~ms}$ duration, rejection factor decreases and appears angle rate that goes with linear acceleration during high shock. Figure 5 shows responses of three channels ( $\underline{X}, \underline{Y}$ and differential ones) of differential CVG under 5 shocks along IA. As can be seen from the peak response values indicated on the figure 5 rejection factor, calculated by (5), is close to $\underline{R}_{s p}=2$.

Let's analyze response to shock in detail. Figure 6 shows four signals responses $(\underline{X}, \underline{Y},(\underline{X}-\underline{Y}) / 2$ and $(\underline{X}+\underline{Y}) / 2)$ of differential CVG to the first of five shocks along gyro IA. Due to presence of angle rate during shock, $\underline{X}$ and $\underline{Y}$ channels have different amplitudes.

To the equal error signals caused by shock, angle rate is added to $\underline{X}$ channel signal and it is subtracted from $\underline{Y}$ channel signal. The difference channel, $(\underline{X}-\underline{Y}) / 2$, presents angle rate acting during shock, and fourth signal $(\underline{X}+\underline{Y}) / 2$, in accordance with (4) does not contain angle rate and presents error signal caused by sensor deformation during shock. 

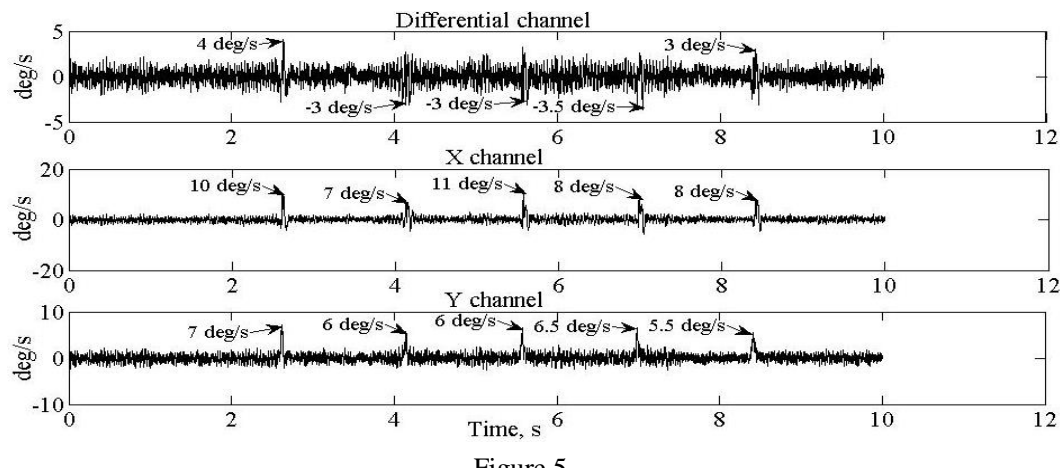

Figure 5

Responses to five shocks of $20 \mathrm{~g}$ amplitude and $2 \mathrm{~ms}$ duration along IA

From Figure 6 one can see that peak value of error signal is about 2 times greater, than that of angle rate. Therefore, if we integrate $\underline{X}$ or $\underline{Y}$ signal to calculate angle error accumulated during shock, it is very important parameter for gyro application in stabilization system operating in harsh environment, then much higher angle error can be obtained in comparison with integration of differential signal.

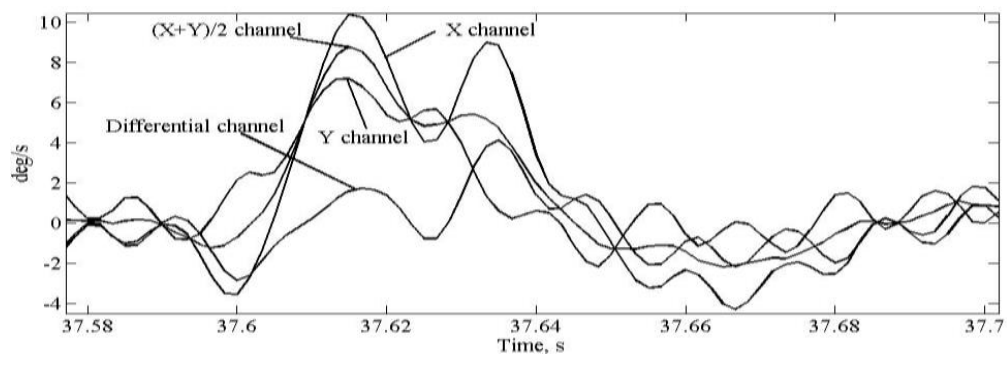

Figure 6

Four signal responses to first of five $20 \mathrm{~g}$ shocks along IA

Figure 7 shows angle errors obtained by integration of $\underline{X}, \underline{Y}$ and differential channel signals during 5 shocks along IA. Differential channel angle error is about 5 times less, than that of $\underline{Y}$ rate channel, and is no more than $2 \operatorname{arc}$ min. Thus, shock rejection factor in terms of angle error for differential $\mathrm{CVG}$ increases to $\underline{R}_{s}=5$ in comparison with rate gyro for $20 \mathrm{~g}$ shock along IA.

Important error component arising under shock is a bias change before and after shock. Figure 8 shows change in biases after each of five of $20 \mathrm{~g}$ shocks along gyro IA. Shock rejection factor in terms of bias change can be calculated using (5), where $\underline{X}_{i}$ and $\underline{Y}_{i}$ are change of biases of corresponding channels before and after $\underline{i}$-th shock. Average over 5 shocks change of absolute value of biases is $0.0097 \mathrm{deg} / \mathrm{s}$ for differential channel and for minimum of $\underline{X}$ and $\underline{Y}$ channels it is $0.03 \mathrm{deg} / \mathrm{s}$, hence, $\underline{R_{s}}=0.03 / 0.0097 \approx 3$. 


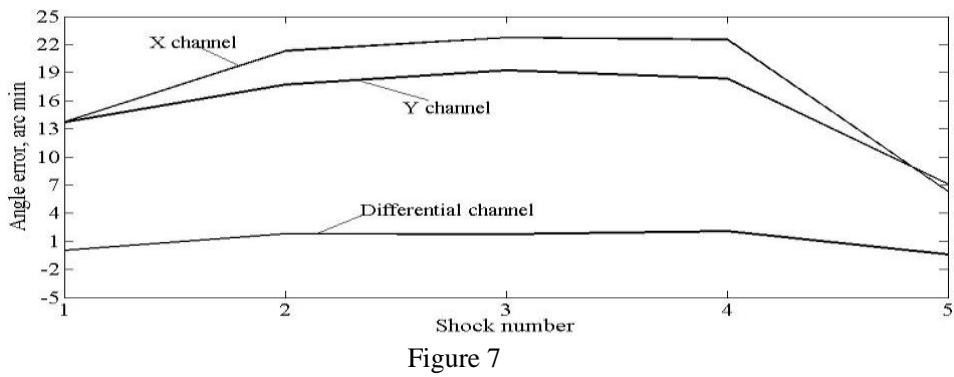

Angle errors during five $20 \mathrm{~g}$ shocks along IA

Using the data presented in Figure 8 bias sensitivity to shock acceleration can also be calculated using (6). Bias sensitivity to shock acceleration for differential channel is about $\underline{S}_{d g}=4.8 * 10^{-4} \mathrm{deg} / \mathrm{s} / \mathrm{g}$, and for minimum of the two ( $\underline{X}$ and $\underline{Y}$ channels) it is about $\underline{S}_{y g}=1.5^{*} 10^{-3} \mathrm{deg} / \mathrm{s} / \mathrm{g}$. Thus, the sensitivity to shock acceleration acting along gyro IA for differential CVG is about 3 times less, than that of rate one.

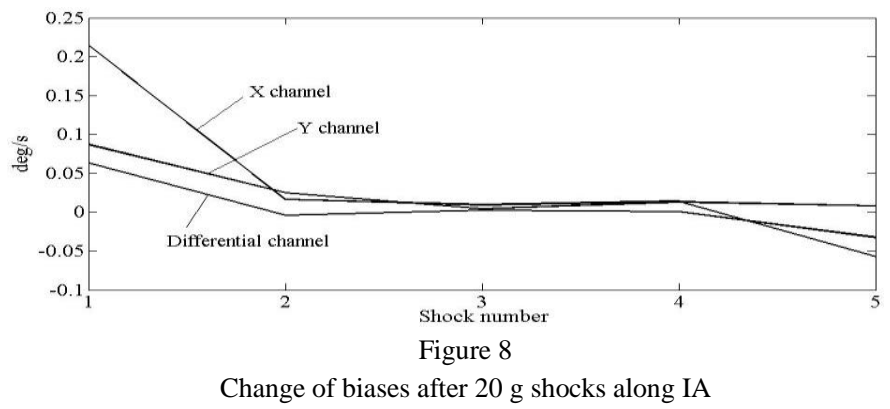

It is expected that higher shocks of $100 \mathrm{~g}$ amplitude and $2 \mathrm{~ms}$ duration, will affect differential CVG channel responses more significant. Figure 9 shows superposed all four channel responses to the first of five of $100 \mathrm{~g}$ shocks along IA. As can be seen $\underline{X}$ channel response is out of measurement range (signal saturation occurs). The same results have been obtained for all 4 successive shocks. Half sum channel signal shows, that sensor deformation caused by $100 \mathrm{~g}$ shock results in equivalent wrong angle rate of more than $100 \mathrm{deg} / \mathrm{s}$. Differential channel shows that angle rate during shock is more than $50 \mathrm{deg} / \mathrm{s}$ versus $4 \mathrm{deg} / \mathrm{s}$ for $20 \mathrm{~g}$ shock. Taking into account that $\underline{Y}$ channel signal amplitude is significantly lower than that of the $\underline{X}$ channel, one can conclude that asymmetry in sensor design and its attachment to the gyro casing resulted in that shock load deformed resonator region close to the $\underline{X}$ sense electrode significantly greater than that of the $\underline{Y}$ electrode. Despite of that designers are trying to design sensor with maximum symmetry (ring, hemisphere and cylinder), residual asymmetry of low-cost sensors remain too great to meet required measurement accuracy during high shock. This problem can be resolved by using dampers or by improving sensor design asymmetry remaining in lowcost category. It is not reasonable to calculate shock rejection factor $\underline{R_{s}}$ in terms of peak values for $100 \mathrm{~g}$ shocks along IA, because of $\underline{X}$ channel signal saturation. 


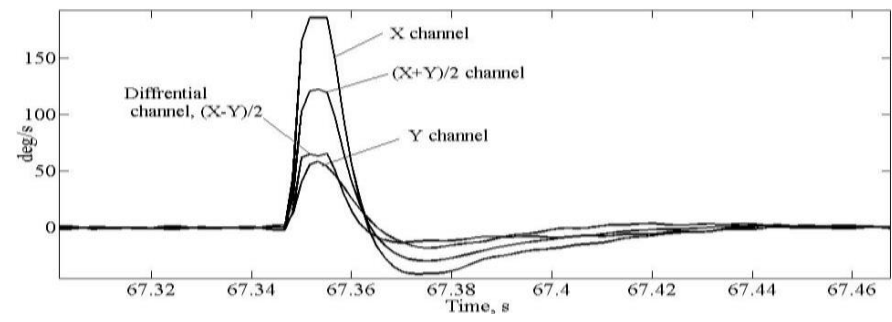

Figure 9

Four signal responses to the first of five $100 \mathrm{~g}$ shocks along IA

Figure 10 shows angle errors of $\underline{X}, \underline{Y}$ and differential channels during each of 5 shocks. Differential channel angle errors for all 5 shocks are almost constant at the level of 5 arc min. It is obvious that some part of these errors are due to $\underline{X}$ channel signal saturation. Nevertheless, shock rejection factor in terms of angle error can be determined at the level of $\underline{R}_{s a} \approx 5$.

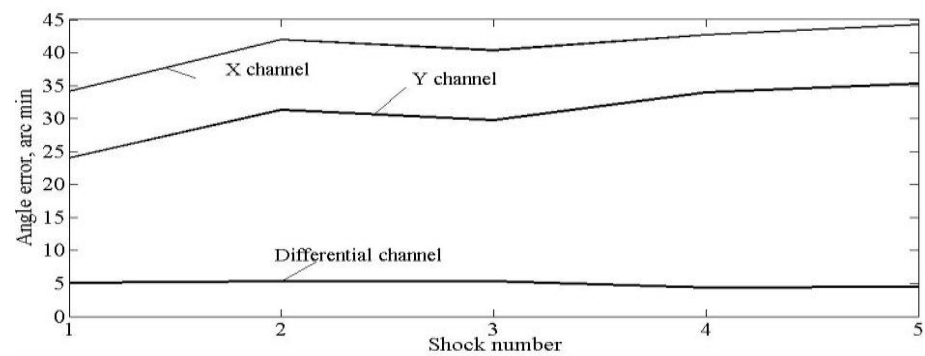

Figure 10

Angle errors during 100g shocks along IA

Shock rejection factor in terms of bias change and differential CVG bias sensitivity to shock acceleration along IA can be calculated, according to (5) and (6), respectively, from the data presented in Figure 11.

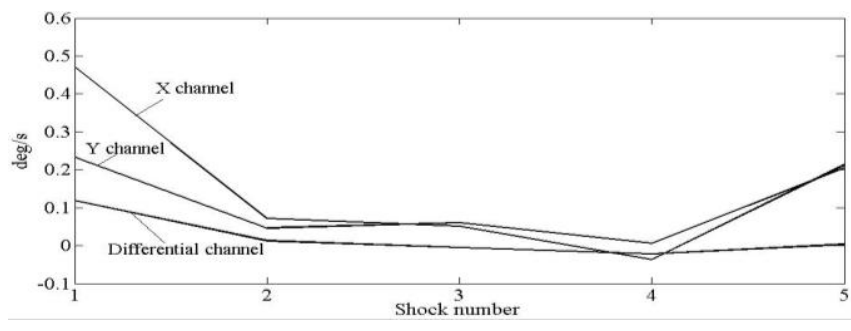

Figure 11

Bias change after five $100 \mathrm{~g}$ shocks along IA

Average over 5 shocks absolute value of bias change for $\underline{X}$ channel is $0.171 \mathrm{deg} / \mathrm{s}$, for $\underline{Y}$ channel it is $0.111 \mathrm{deg} / \mathrm{s}$ and for differential channel it is $0.033 \mathrm{deg} / \mathrm{s}$, hence, $\underline{R}_{\underline{s}}=0.111 / 0.033 \approx 3.4$. Differential $\mathrm{CVG}$ bias sensitivity to shock acceleration along IA is $0.033 / 100=3.3 * 10^{-4} \mathrm{deg} / \mathrm{s} / \mathrm{g}$. The same parameter under $20 \mathrm{~g}$ shock, 
obtained above, is $4.8 * 10^{-4} \mathrm{deg} / \mathrm{s} / \mathrm{g}$, this difference is supposedly due to $\underline{X}$ channel signal saturation under $100 \mathrm{~g}$ shocks.

\subsection{Lateral Shocks}

Figure 12 shows $\underline{X}, \underline{Y}$ and differential channel responses to 5 lateral shocks of $20 \mathrm{~g}$ amplitude and $2 \mathrm{~ms}$ duration. Calculation of rejection factor in terms of peak value over 5 shock using (5) results in $\underline{R}_{s p} \approx 2$. Thus, rejection factors for lateral and along IA $20 \mathrm{~g}$ amplitude shocks are equal to each other.

Almost the same behavior of angle error for differential channel is observed during lateral shocks of $20 \mathrm{~g}$ amplitude, shown in Figure 13, but in this case shock rejection factor in terms of angle error for differential mode of operation increases to $\underline{R}_{s a}=6$ in comparison with rate mode, with no more, than 2 arc min angle error. Real gyro turn angle after shock is zero. This turn angle is monitored by an optical method.

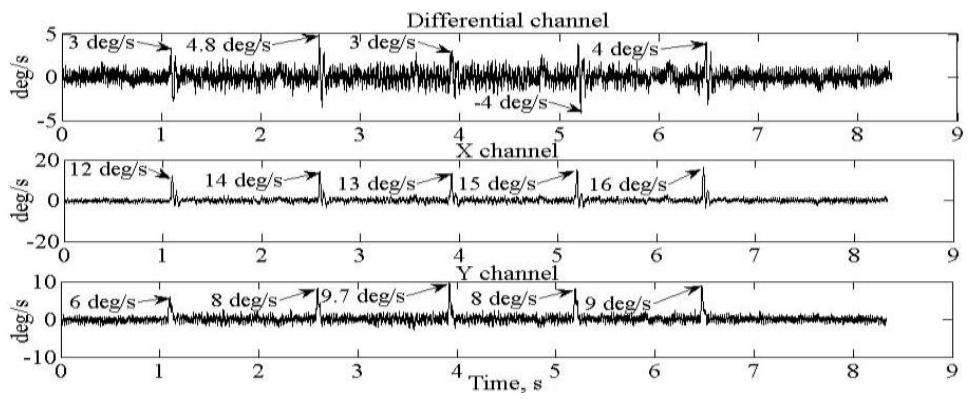

Figure 12

Responses to five lateral shocks of $20 \mathrm{~g}$ amplitude

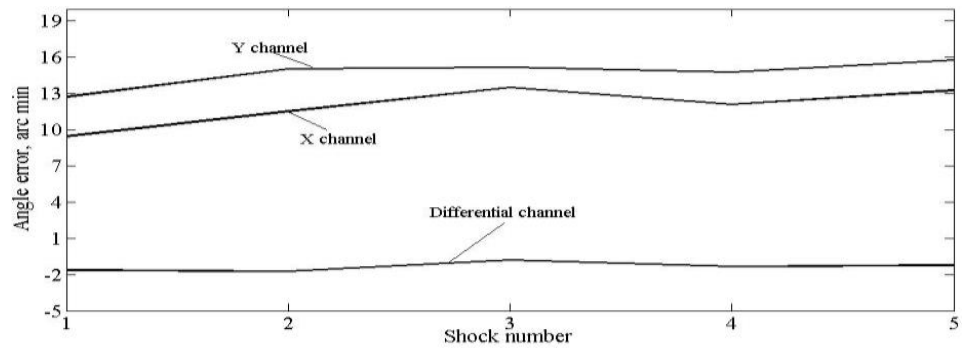

Figure 13

Angle errors during $20 \mathrm{~g}$ lateral shocks

Figure 14 shows change of biases after lateral shocks. In this case rejection factor in terms of bias change is greater than that of along IA and evaluated, using data presented in figure 14, as $\underline{R}_{\underline{s}} \approx 8$. Differential channel bias sensitivity to lateral shock acceleration is about $\underline{\mathrm{S}}_{\mathrm{g} d}=10^{-3} \mathrm{deg} / \mathrm{s} / \mathrm{g}$ versus $\underline{Y}$ channel sensitivity which is evaluated as $\underline{S}_{g y}=8 * 10^{-3} \mathrm{deg} / \mathrm{s} / \mathrm{g}$. 


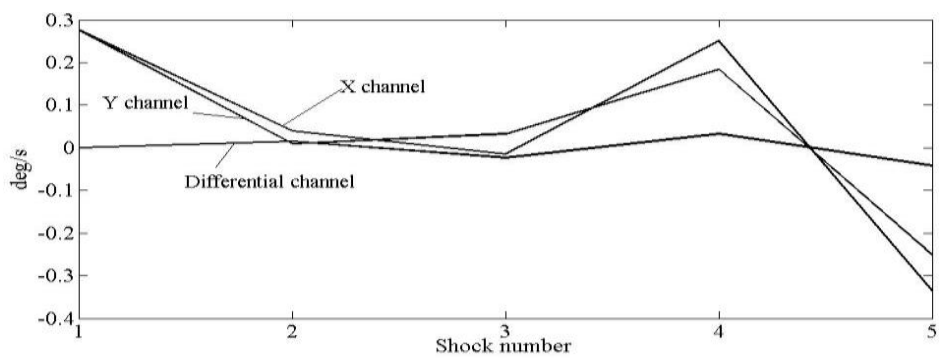

Figure 14

Bias change after $20 \mathrm{~g}$ lateral shocks

Figure 15 demonstrates superposed all four channels of differential CVG responses to the first of five of $100 \mathrm{~g}$ lateral shocks. Error signal peak value presented by $(\underline{X}+\underline{Y}) / 2$ channel is about 3.5 time higher, than angle rate signal presented by differential channel.

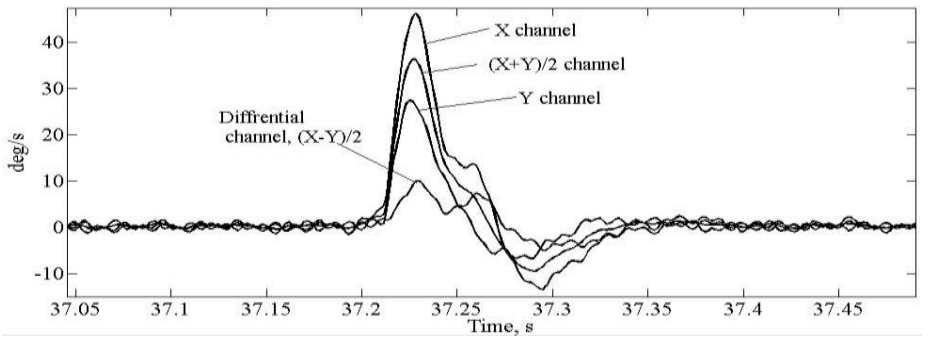

Figure 15

Four signal responses to the first of five $100 \mathrm{~g}$ lateral shocks

It should be noted that in this case there is no saturation in the measured signals. Figure 16 demonstrates responses to five lateral shocks of $100 \mathrm{~g}$ amplitude for
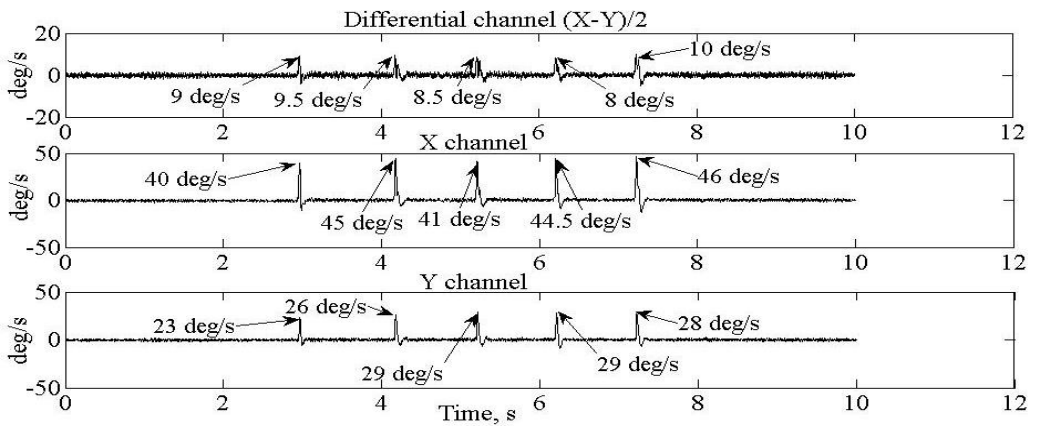

Figure 16

Responses to five $100 \mathrm{~g}$ lateral shocks

each of three differential CVG signals with indication of peak values for each of them. $\underline{X}$ and $\underline{Y}$ channel peak values do not always coincide with the differential 
channel peak because peak values do not always coincide in time. Calculation using (5) yields rejection factor $\underline{R}_{s p} \approx 3$ for lateral $100 \mathrm{~g}$ shocks.

Figure 17 shows angle errors of $\underline{X}, \underline{Y}$ and differential channels during each of 5 lateral shocks. Differential channel maximum angle error reaches 18 arc min, and rejection factor for lateral shock of $100 \mathrm{~g}$ amplitude is $\underline{R}_{s a} \approx 2.5$.

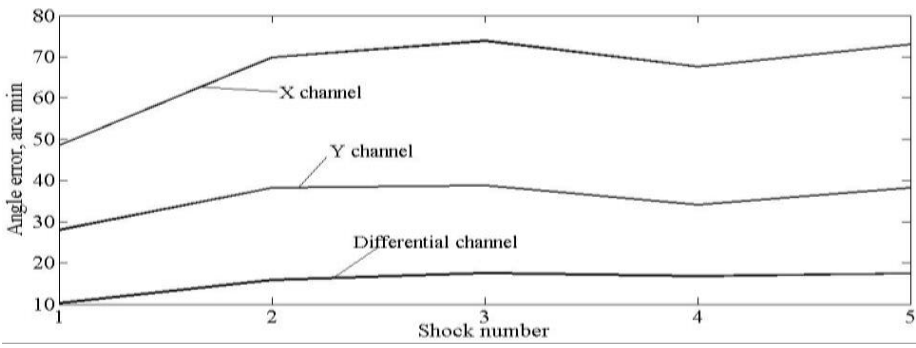

Figure 17

Angle errors during five $100 \mathrm{~g}$ lateral shocks

Figure 18 presents bias change after each of five lateral shocks of $100 \mathrm{~g}$ amplitude and $2 \mathrm{~ms}$ duration. Shock rejection factor in terms of bias change and differential CVG bias sensitivity to lateral shock acceleration yield $\underline{R}_{s b} \approx 4$ and $3 * 10^{-4} \mathrm{deg} / \mathrm{s} / \mathrm{g}$, respectively.

Let's summarize shock tests in the Table 1. Table 1 data show that differential CVG shock rejection factor is minimum, 2 times greater, than that of rate CVG in terms of peak values. In terms of angle error shock rejection factor for differential CVG is minimum, 2.5 times greater for $100 \mathrm{~g}$ lateral shocks and is 6 times greater for $20 \mathrm{~g}$ shocks, than that of rate CVG.

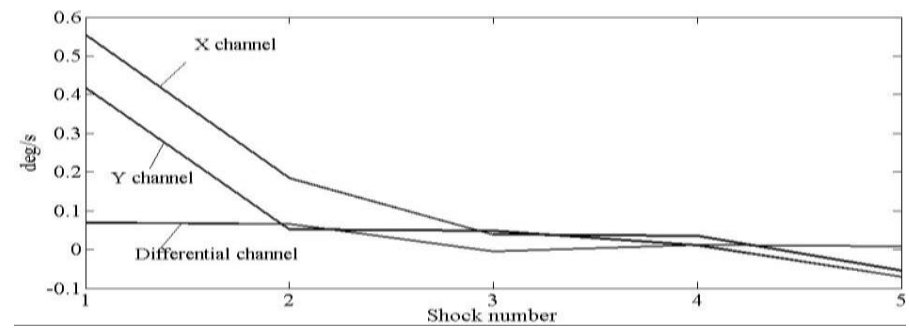

Figure 18

Bias change after five $100 \mathrm{~g}$ lateral shocks

As to shocks along IA rejection factor in terms of angle error is 5 times greater for both $20 \mathrm{~g}$ and $100 \mathrm{~g}$ shocks, than that of for rate CVG. Shock rejection factor in terms of bias change before and after shocks is minimum, 3 times greater for both $20 \mathrm{~g}$ and $100 \mathrm{~g}$ shocks along IA and 4 times greater for lateral shocks, than that of for rate $\mathrm{CVG}$. 
Table 1

Summarized data on shock test results

\begin{tabular}{|c|c|c|c|c|c|c|c|}
\hline \multicolumn{2}{|c|}{$\begin{array}{l}\text { Rejection factor in } \\
\text { terms of peak value } \\
\qquad \underline{R_{p}}\end{array}$} & \multicolumn{2}{|c|}{$\begin{array}{l}\text { Rejection factor in } \\
\text { terms of angle error } \\
\quad \underline{R}_{a} \text { max error } \\
\quad(\operatorname{arc~min})\end{array}$} & \multicolumn{2}{|c|}{$\begin{array}{c}\text { Rejection factor in } \\
\text { terms of bias change } \\
\underline{R_{b}} \text { max bias change } \\
\text { (deg/s) }\end{array}$} & \multicolumn{2}{|c|}{$\begin{array}{c}\text { Diff. CVG bias } \\
\text { sensitivity to shock } \\
\text { acceleration, } \\
\text { deg/s/g }\end{array}$} \\
\hline \multicolumn{8}{|c|}{ Shock amplitude $20 \mathrm{~g}, 2 \mathrm{~ms}$ duration } \\
\hline $\begin{array}{c}\text { Along } \\
\text { IA }\end{array}$ & Lateral & $\begin{array}{c}\text { Along } \\
\text { IA }\end{array}$ & Lateral & $\begin{array}{c}\text { Along } \\
\text { IA }\end{array}$ & Lateral & $\begin{array}{c}\text { Along } \\
\text { IA }\end{array}$ & Lateral \\
\hline 2 & 2 & $\begin{array}{l}5 \\
2 \\
\end{array}$ & $\begin{array}{l}6 \\
2 \\
\end{array}$ & $\begin{array}{c}3 \\
0.06\end{array}$ & $\begin{array}{c}8 \\
0.03\end{array}$ & $4.8 * 10^{-4}$ & $10^{-3}$ \\
\hline \multicolumn{8}{|c|}{ Shock amplitude $100 \mathrm{~g}, 2 \mathrm{~ms}$ duration } \\
\hline saturation & 3 & $\begin{array}{l}5 \\
5\end{array}$ & $\begin{array}{l}2.5 \\
18\end{array}$ & $\begin{array}{c}3.4 \\
0.12 \\
\end{array}$ & $\begin{array}{c}4 \\
0.07\end{array}$ & $3.3 * 10^{-4}$ & $3 * 10^{-4}$ \\
\hline
\end{tabular}

\section{Vibration Sensitivity}

Gyroscopes in most applications operate on moving vehicles and they are subjected to vibrations during motion and at stops when engine is running. Gyros change their biases and other parameters under vibration. The main reason of this is also the design asymmetry discussed in the previous section. In many low-cost gyros bias change under vehicle vibration reduces measurement accuracy much greater, than in absence of vibration. Gyro sensitivity to linear vibration expressed in $g$ units is no less important parameter from practical point of view, than bias stability in absence of vibration which value one can see in any gyro data sheet.

\subsection{Vibration along IA}

\subsection{1 $\quad g$-Dependent Bias}

Figure 19 shows $\underline{X}, \underline{Y}$ and differential channel biases change versus amplitude of sinusoidal vibration for 50,100 and $300 \mathrm{~Hz}$ vibration frequencies. Bias change is calculated as difference between corresponding channel bias obtained at vibration and the same channel bias at no vibration. As can be seen from this figure the higher the frequency of vibration, the greater the change of the bias, especially for higher vibration amplitude. For $3 g$ vibration amplitude and $300 \mathrm{~Hz}$ frequency change of biases for $\underline{X}$ and $\underline{Y}$ channels are almost equal to each other at a value of $1.2 \mathrm{deg} / \mathrm{s}$ and for differential channel change of the bias is about $0.1 \mathrm{deg} / \mathrm{s}$. So, change of the bias for differential channel 12 times less than that of for rate channels. As can visually be estimated from Figure 19 at any frequency and amplitude of vibration, in the considered here ranges, the bias change of differential channel is less than that of the $\underline{X}$ and $\underline{Y}$ channels of about 10 times. 


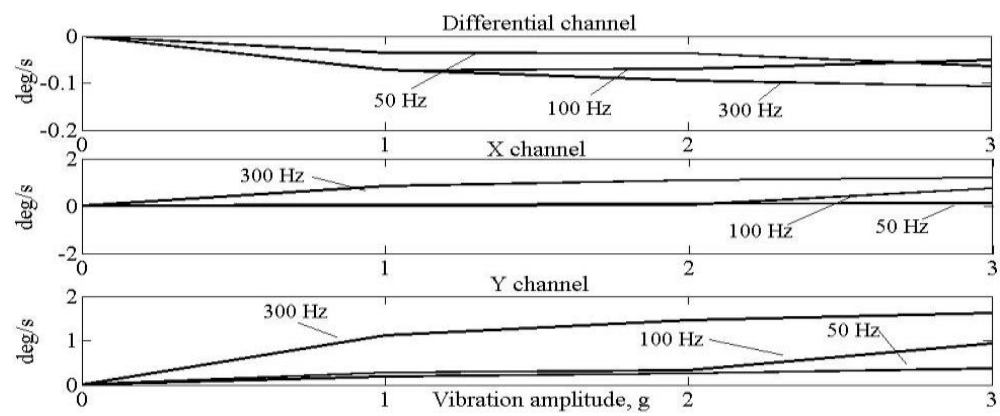

Figure 19

Bias change under sinusoidal vibration of different frequencies

Figure 20 shows bias sensitivities of $\underline{X}, \underline{Y}$ and differential channels to vibration amplitude versus frequency. These sensitivities have been calculated as a tangent of tilt angles of least squares straight line drawn by the data presented in Figure 19. As a result the bias sensitivities to vibration amplitude in $\mathrm{deg} / \mathrm{s} / \mathrm{g}$ within the range of [1 3] $\mathrm{g}$ for each of three vibration frequencies are obtained and graphed in Figure 20.

One can see from this graph that bias sensitivity to vibration amplitude is dependent on frequency. So, it will be difficult to calibrate this parameter using accelerometer measurement data, as it is often made in low-cost inertial measurement units [18], [13] and has been noted in [9]. Differential channel sensitivity to vibration at $300 \mathrm{~Hz}$ is $\underline{S}_{d g, 300}=0.034 \mathrm{deg} / \mathrm{s} / \mathrm{g}$ and for $\underline{X}$ and $\underline{Y}$ channels they are $\underline{S}_{x g, 300}=0.38 \mathrm{deg} / \mathrm{s} / \mathrm{g}$ and $\underline{S}_{y g, 300}=0.52 \mathrm{deg} / \mathrm{s} / \mathrm{g}$, respectively. It is about an order of magnitude greater, than for differential channel. Besides, using data presented in Figure 20, bias sensitivity to vibration amplitude and frequency can be calculated by the same technique with the aid of least squares straight line.

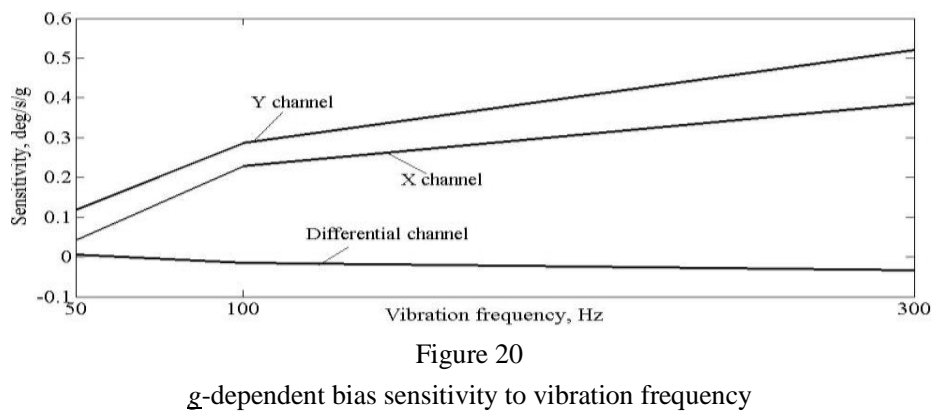

For differential CVG bias sensitivity to vibration amplitude and frequency is $\underline{S}_{d, g, f}=1.3 * 10^{-4} \mathrm{deg} / \mathrm{s} / \mathrm{g} / \mathrm{Hz}$. In the frequency range far from $\mathrm{CVG}$ resonant frequency it is reasonable to suppose that parameter $\underline{S}_{d, g}$ will be changed almost linearly versus frequency, as it is in the range of up to $300 \mathrm{~Hz}$, hence, one can make a linear prediction of differential CVG bias change. 


\subsection{2 $\quad g$-Dependent Noise}

Figure 21 presents differential (left), $\underline{X}$ and $\underline{Y}$ (right) channels root of Allan variances for different vibration amplitudes at frequency $100 \mathrm{~Hz}$. Figure 21 demonstrates that such noise components as white noise and random walk almost do not depend on vibration amplitude, whereas bias instability and rate random walk are g-dependable. Because bias instability is the most important gyro parameter we will focus on g-sensitivity of this noise component, but firstly let's discuss relationship between RMS values of $\underline{X}, \underline{Y}$ and differential channels total noises.
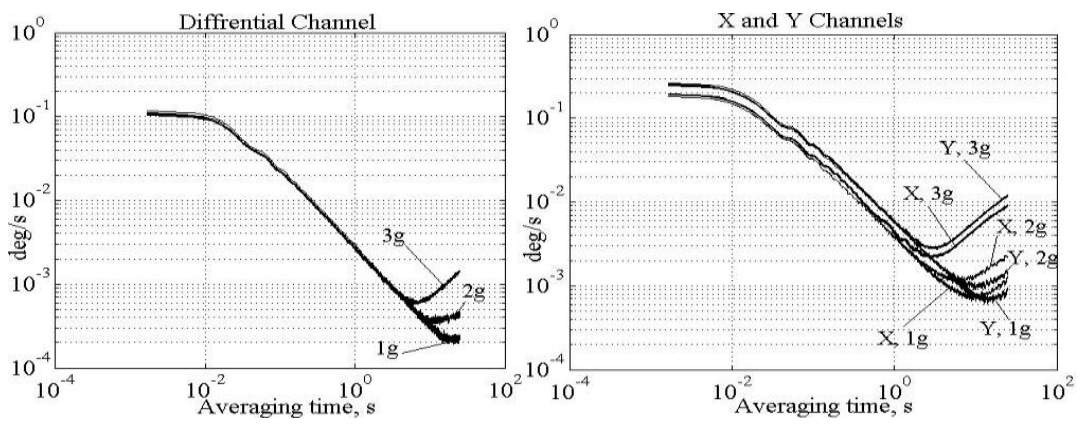

Figure 21

Differential CVG signals root of Allan variances at vibration frequency $100 \mathrm{~Hz}$

From Figure 21 one can see that all noise components (white noise, random walk, bias instability and rate random walk) of differential channel are appreciably less than those of for $\underline{X}$ and $\underline{Y}$ channels. The same results are obtained for $50 \mathrm{~Hz}$ and $300 \mathrm{~Hz}$ vibration frequencies. This means that total noise RMS value for differential channel signal is less, than that of for $\underline{X}$ and $\underline{Y}$ channels. There is well known relationship between standard deviations of the considered channels that follows from the expression $d=(\underline{X}-\underline{Y}) / 2$, where $d$ is differential channel signal

$\sigma_{d}=\frac{1}{2} \sqrt{\sigma_{x}^{2}+\sigma_{y}^{2}-2 \operatorname{cov}(X, Y)}$

Where, $\sigma_{d}, \sigma_{x}, \sigma_{\mathrm{y}}$ are RMS values of noises for differential, $\underline{X}$ and $\underline{Y}$ channels, respectively; $\operatorname{cov}(\underline{X}, \underline{Y})$ is $\underline{X}$ and $\underline{Y}$ inter-channel covariance. If inter-channel covariance is positive and $\sigma_{x} \approx \sigma_{y}$, then $\sigma_{d}$ is less than $\sigma_{x}$ and $\sigma_{y}$. Inter-channel covariance in deferential CVG is dependent on standing wave drive technique and its value changes versus external disturbances. Figure 22 shows inter-channel correlation coefficients (covariance normalized by $\sigma_{x}$ and $\sigma_{y}$ ) when there is absent and present external vibration with different parameters. All values of correlation coefficients are positive and sufficiently large, as a consequence, $\sigma_{d}$ is less than minimum of $\sigma_{x}$ and $\sigma_{y}$ up to 2.8 times.

Let's now determine g-sensitivity of bias instability. Figure 23 shows dependence of bias instability versus vibration amplitude. Sensitivity of bias instability to vibration amplitude is calculated as a tangent of tilt angles of least squares straight 


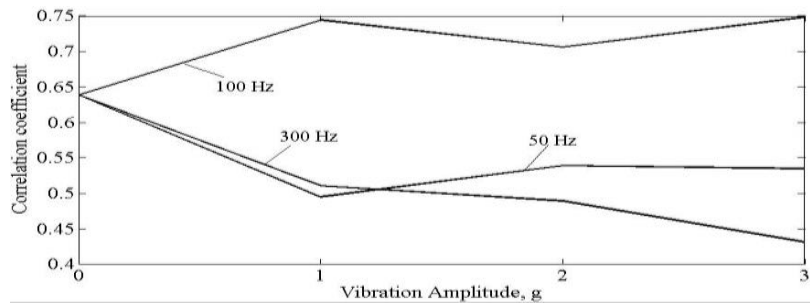

Figure 22

Inter-channel correlation coefficients for different vibration parameters

line drawn by the data presented in Figure 23 for each of three vibration frequencies. Calculation results are presented in the Table 2.

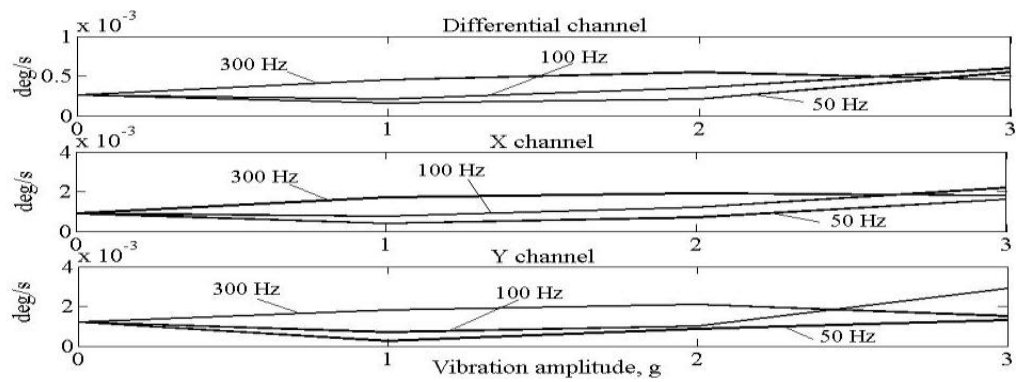

Figure 23

Bias instability for different vibration parameters

Table 2

g-sensitivity of bias instability during vibration along IA

\begin{tabular}{|c|c|c|c|}
\hline $\begin{array}{c}\text { Vibration } \\
\text { frequency, Hz }\end{array}$ & $\begin{array}{c}\text { Differential channel bias } \\
\text { g-sensitivity, deg/s/g }\end{array}$ & $\begin{array}{c}\underline{X} \text { channel bias g- } \\
\text { sensitivity, deg/s/g }\end{array}$ & $\begin{array}{c}\underline{Y} \text { channel bias g- } \\
\text { sensitivity, deg/s/g }\end{array}$ \\
\hline 50 & $9.2^{*} 10^{-5}$ & $2.4^{*} 10^{-4}$ & $9 * 10^{-5}$ \\
\hline 100 & $1.16^{*} 10^{-4}$ & $4.35^{*} 10^{-4}$ & $5.4 * 10^{-4}$ \\
\hline 300 & $6.7 * 10^{-5}$ & $2.9^{*} 10^{-4}$ & $10^{-4}$ \\
\hline Mean & $9.2^{-4} 10^{-5}$ & $3.2^{*} 10^{-4}$ & $2.4^{*} 10^{-4}$ \\
\hline
\end{tabular}

Mean value of bias g-sensitivity for differential channel is 2.6 times less than a minimum of that for $\underline{X}$ and $\underline{Y}$ rate channels.

\subsection{Lateral Vibration}

The behavior of noise components during lateral vibration in the same ranges of vibration parameters is similar to that of along IA. So, we present lateral vibration tests in the resultant Table 3. Differential channel g-sensitivity mean value of bias instability is 1.7 times less than the minimum of that for $\underline{X}$ and $\underline{Y}$ rate channels. Inter-channel correlation coefficient for lateral vibration is also positive and it is in the range of $0.43-0.75$. 
Table 3

g-sensitivity of bias instability during lateral vibration

\begin{tabular}{|c|c|c|c|}
\hline $\begin{array}{c}\text { Vibration } \\
\text { frequency, Hz }\end{array}$ & $\begin{array}{c}\text { Differential channel } \\
\text { bias } g \text {-sensitivity, deg/s/g }\end{array}$ & $\begin{array}{c}\underline{X} \text { channel bias } g- \\
\text { sensitivity, deg/s/g }\end{array}$ & $\begin{array}{c}\underline{Y} \text { channel bias } g- \\
\text { sensitivity, deg/s/g }\end{array}$ \\
\hline 50 & $8.5^{*} 10^{-5}$ & $3.75 * 10^{-4}$ & $2.95 * 10^{-4}$ \\
\hline 100 & $5.4^{-4} 10^{-4}$ & $1.75 * 10^{-3}$ & $9.3 * 10^{-4}$ \\
\hline 300 & $5.25 * 10^{-4}$ & $2.04 * 10^{-3}$ & $7.1 * 10^{-4}$ \\
\hline Mean & $3.8^{-4} 10^{-4}$ & $1.4 * 10^{-3}$ & $6.5^{*} 10^{-4}$ \\
\hline
\end{tabular}

For these vibration parameters differential channel RMS value $\sigma_{d}$ of total noise is 2.2 times less than minimum of $\sigma_{x}$ and $\sigma_{y}$.

\section{Conclusion}

Differential CVG can be considered as a third mode of operation for a vibratory gyro. This mode of operation can be called an intelligent robustness enhancement to external disturbances one. A triple-mode gyro can be implemented for both MEMS and non-MEMS vibratory gyros using an intelligent algorithm for choosing a corresponding mode of operation in accordance with changing environmental conditions. In addition, it was found that the bias sensitivity to the amplitude of external vibrations is dependent on vibration frequency. It was also found that differential single mass CVG output noise is less than that of the rate CVG for both present and absent of external vibrations. The results obtained in this paper can be extended to MEMS gyros and have many important applications in intelligent systems.

\section{References}

[1] Alireza Namadmalan, Javad Shokrollahi Moghani: New Resonant Inverter Tuning for Three-Phase Current Source Parallel Resonant Inverters, Acta Polytechnica Hungarica, v.11, \#5, pp. 217-234, 2014

[2] A. Jeanroy, P. Featonby, J-M. Caron: Low-Cost Miniature and Accurate Sensors for Tactical Applications, 10-th S. Petersburg Int. Conf. on Integrated Navigation Systems, pp. 286-293, May, 2003

[3] A. A. Trusov, A. R. Schofield, A. M. Shkel: Micromachined tuning fork gyroscopes with ultra-high sensitivity and shock rejection, US Patent \#8322213, publ. date 4 Dec. 2011

[4] B. Gallacher, Zh. Hu, S. Bowles: Full control and compensation scheme for a rate-integrating MEMS gyroscope, $13^{\text {th }}$ Int. Conf. on Dynamical Systems - Theory And Applications, Lodz, Poland, paper id: ENG267, Dec. 7-10, 2015

[5] Chen Fan, Xiaoping Hu, Xiaofeng He, Kanghua Tang, Bing Luo: Observability Analysis of a MEMS INS/GPS Integration System with Gyroscope G-Sensitivity Errors, Sensors, 14, pp. 16003-16016, Sept., 2014. doi:10.3390/s140916003 
[6] D. D. Lynch: Coriolis Vibratory Gyroscope, IEEE Standard Specification Format Guide and Test Procedure for Coriolis Vibratory Gyros, IEEE std.1431 ${ }^{\mathrm{TM}}$, Annex B, pp. 56-66, Dec. 2004

[7] D. D. Lynch, A. Matthews: Dual Mode Hemispherical Resonator Gyro Operating Characteristics, 3-rd S. Petersburg Int. Conf. on Integrated Navigation Systems, part 1, pp. 37-44, May 1996

[8] David M. Rozelle: Hemispherical Resonator Gyro: From Wineglass to the Planets, http://www.northropgrumman.com/capabilities/hrg/documents/hrg.pdf

[9] H. Weinberg: Gyro Mechanical Performance: The Most Important Parameter, Analog Devices Inc., Technical article MS-2158, pp. 1-5, Sept. 2011, www.analog.com

[10] J. A. Gregory: Characterization Control and Compensation of MEMS Rate and Rate-Integrating Gyroscopes".- Ph.D. Dissertation, Michigan University, P.198, 2012

[11] J. Y. Cho: High-Performance Micromachined Vibratory Rate- And RateIntegrating Gyroscopes, Ph.D. Dissertation, Michigan University, P.293, 2012

[12] J-K. Woo, J. Y. Cho, Ch. Boyd, Kh. Najafi: Whole-Angle-Mode Micromachined Fused-Silica Birdbath Resonator Gyroscope (Wa-Brg), IEEE MEMS Conf., San Francisco, CA, USA, January 26-30, 2014

[13] J. B. Bancroft and G. Lachapelle: Estimating MEMS Gyroscope GSensitivity Errors in Foot Mounted Navigation, $2^{\text {nd }}$ Int. Conf. on Ubiquitous Positioning, Indoor Navigation and Location-Based Service, Helsinki, Finland, pp. 1-6, 2-5 Oct 2012

[14] P. Soobramaney: Mitigation of the Effects of High Levels of HighFrequency Noise on MEMS Gyroscopes, Ph.D. Dissertation, Auburn University, Alabama, p. 200, 3 Aug. 2013

[15] R. Schofield, A. A. Trusov, A. M. Shkel: Multi-Degree of Freedom Tuning Fork Gyroscope Demonstrating Shock Rejection, IEEE Conf. on Sensors, Atlanta, Georgia, USA, pp. 120-123, 28-31 Oct. 2007

[16] S. Dellea, F. Giacci, P. Rey, A. Capodici, G. Langfelder: Reliability of gyroscopes based on piezoresistive nano-gauges against shock and freedrop tests, $29^{\text {th }}$ IEEE Int. Conf. on Micro Electro Mechanical Systems (MEMS), Shanghai, China, pp. 255-258, 24-28 Jan. 2016

[17] V. V. Chikovani, E. O. Umakhanov, P. I. Marusyk: The compensated differential CVG, Gyro Technology Symposium, Germany, Karlsruhe university, pp. 3.1-3.8, 16-17 Sept. 2008 
[18] V. V. Chikovani: Method of angle rate measurement with Coriolis vibratory gyroscope, UA Pat. 95709 Ukraine, Icl. G01 C 19/02, Publ. date 25 Aug. 2011 (bul. \# 16/2011 in Ukrainian)

[19] V. V. Chikovani, O. A. Suschenko: Differential mode of operation for ringlike resonator CVG, IEEE Proc. Intern. Conf. on Electronics and Nanotechnology, Kyiv, Ukraine, pp. 451-455, 15-18 April 2014

[20] V. V. Chikovani, G. V. Tsiruk: Bias Compensation in Differential Coriolis Vibratory Gyro, Electronics and control systems, NAU, Kyiv, Ukraine, \#4 (38), pp. 99-103, 2013

[21] V. V. Chikovani, H. V. Tsiruk: Differential Mode of Operation For Multimode Vibratory Gyroscope, IEEE Proc. Intern. Conf. on Actual Problem of Unmanned Aerial vehicles Development (APUAVD), NAU, Kyiv, Ukraine, pp. 87-90, Oct. 13-15, 2015

[22] Zhong Su, Ning Liu, Qing Li, Mengyin Fu, Hong Liu, Junfang Fan: Research on the Signal Process of a Bell-Shaped Vibratory Angular Rate Gyro, Sensors, 14, 5254-5277, 2014; doi: 10.3390/s140305254 